\title{
Urolitíase em cães: avaliação quantitativa da composição mineral de 156 urólitos
}

\author{
Canine urolithiasis: quantitative evaluation of mineral composition of 156 uroliths
}

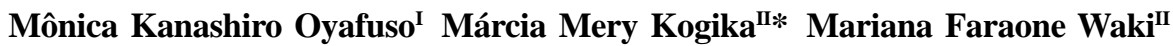 \\ Christiane Seraphim Prosser ${ }^{\mathrm{I}}$ Carolina Zaghi Cavalcante ${ }^{\mathrm{I}}$ \\ Vera Assunta Batistini Fortunato Wirthl ${ }^{\mathrm{II}}$
}

\section{RESUMO}

O estudo teve como objetivo avaliar os casos de urolitíase canina em que a composição mineral dos urólitos foi analisada quantitativamente. Foi avaliada quantitativamente a composição mineral de 156 urólitos obtidos de cães (nefrólitos, ureterólitos, urocistólitos e uretrólitos). Desse total, $79,5 \%(n=124)$ eram simples, $18 \%(n=28)$ eram compostos e apenas $2,5 \%(n=4)$ eram mistos. A estruvita foi $o$ tipo mineral mais frequente nos urólitos simples $(47,6 \% ; n=59)$, em todos os mistos $(100 \% ; n=4)$ e nas camadas núcleo e pedra de urólitos compostos (32,1 e 75\%, respectivamente). O oxalato de cálcio foi o segundo mineral mais frequente dos urólitos simples $(37,9 \%, n=47)$. Ao contrário do que é preconizado para os urólitos simples, as recomendações para o tratamento de urólitos compostos são mais complexas, tais como protocolos de tratamento de dissolução diferentes (se composto por minerais distintos e passíveis de dissolução como urato e estruvita). Além disso, a dissolução pode não ser viável, caso ocorra presença de material insolúvel envolvendo o urólito ou se este representar mais de $20 \%$ da camada. Vinte e dois urólitos compostos $(78,7 \%)$ apresentaram uma camada externa não passível de dissolução (oxalato de cálcio ou fosfato de cálcio); dois $(7,1 \%)$ apresentaram camadas externas passíveis de dissolução (estruvita ou urato), porém camadas mais internas não solúveis, o que permitiria apenas a dissolução parcial do urólito. Assim, o conhecimento da composição de todas as camadas que compõem o urólito é essencial para o entendimento da formação do cálculo e consequentemente para a indicação do tratamento adequado, assim como para prevenção de recidivas.

Palavras-chave: cálculo urinário, composição mineral, análise quantitativa.

\begin{abstract}
The aim of this study was to evaluate dogs with urolithiasis in which mineral composition of calculi was quantitatively analyzed. Quantitative mineral composition was performed in 156 canine uroliths. Simple uroliths represented $79.5 \%(n=124)$ of the cases, $18 \%$ were compound $(n=28)$ and only $2.5 \%(n=4)$ of the calculi were mixed. Struvite was the most frequent mineral type of simple uroliths $(47.6 \% ; n=59)$ as well as in all mixed $(100 \% ; n=4)$ and in the core and stone uroliths (32.1\% and $75 \%$, respectively). Calcium oxalate was the second more frequent mineral composition of simple uroliths (37.9\%; $n=47)$. Unlike simple uroliths, recommendation for the treatment of compound uroliths is more complex, and diet protocols for calculi dissolution may be different when the calculus is composed by different minerals that are possible to be dissolved (e.g. urate and stuvite). Besides, dissolution may not be feasible if it occurs in presence of insoluble material involving urolith or if it represents more than $20 \%$ of the layer. Twenty two compound uroliths (78.7\%) presented an external layer that was not possible to be dissolved (calcium oxalate or calcium phosphate); two calculi (7.1\%) had superficial layers dissolvable (struvite or urate), but inner layers were not soluble, which allowed only partial dissolution of urolith. Knowledge of all urolith layers mineral composition is essential for the understanding of calculus formation and for the adequate treatment indication as well as for the procedures to prevent recurrence.
\end{abstract}

Key words: urinary calculus, mineral composition, quantitative analysis.

IPrograma de Pós-graduação em Clínica Veterinária, Universidade de São Paulo (USP), São Paulo, SP, Brasil.

IIDepartamento de Clínica Médica, Faculdade de Medicina Veterinária e Zootecnia (FMVZ), USP. Av. Prof. Orlando Marques de Paiva, 87, 05508-270, São Paulo, SP, Brasil. E-mail: mmkogika@usp.br. *Autor para correspondência.

IIIHospital Veterinário, FMVZ, USP, São Paulo, SP, Brasil. 


\section{INTRODUÇÃO}

O sistema urinário de animais terrestres tem habilidade de formar urina hiperosmolar (concentrada), sendo uma de suas funções a eliminação de resíduos na forma líquida (SENIOR \& FINLAYSON, 1986). Em certas condições, alguns resíduos, especialmente minerais, precipitam e formam cristais. Se esses cristais permanecerem retidos no sistema urinário, pode haver a combinação com matriz orgânica e/ou outros minerais, promovendo seu crescimento e a formação de cálculos (OSBORNE \& CLINTON, 1986; OSBORNE et al., 1999b).

Com a prevalência relatada entre 0,4 e 2,0\% (BOVEE \& McGUIRE, 1984), a urolitíase é a terceira doença mais frequente do trato urinário de cães (CAMARGO, 2004; LULICH et al., 2004; SOSNAR et al., 2005). Não deve ser entendida como uma doença isolada, mas sim como uma consequência de uma ou mais anormalidades subjacentes (OSBORNE et al., 1989). A interação de fatores de origem familial, congênita ou processos patológicos adquiridos, em combinação, aumenta progressivamente o risco de formação de urólitos (OSBORNE \& CLINTON, 1986; OSBORNE et al., 1989). Dentre esses já conhecidos, destacam-se a raça, o sexo, a idade, as anormalidades anatômicas ou funcionais do trato urinário, as anormalidades metabólicas, as infecções urinárias, a dieta e o $\mathrm{pH}$ da urina. Cada um desses elementos pode ter efeitos diferentes no desenvolvimento ou na prevenção dos diversos tipos de urólitos. O reconhecimento desses riscos auxilia o diagnóstico precoce e permite o planejamento de terapia e de modificações dietéticas que possam minimizar a formação de urólitos e recidivas (OSBORNE et al., 1999b). Segundo OSBORNE et al. (1989), é improvável que apenas a remoção cirúrgica do urólito impeça recidivas. Portanto, a detecção do urólito é apenas o início do processo diagnóstico (LULICH et al., 2004).

Os urólitos podem ser compostos por um ou mais tipos de minerais, que podem ser depositados em lâminas (camadas) ou simplesmente misturados entre si. Além dos cristais biológicos, outros agentes, como metabólitos de fármacos e corpos estranhos (como material de sutura, pelos), podem estar presentes (ULRICH et al., 1996). Dependendo da composição e da distribuição dos componentes minerais, o urólito pode conter as camadas: núcleo (região central), pedra, parede e cristais de superfície. Camadas concêntricas são comuns e representam a evolução de deposição de mineral e matriz, pois está na dependência da composição da urina que varia diariamente (OSBORNE \& CLINTON, 1986; ULRICH et al., 1996).

Assim, baseando-se no tipo e na quantidade mineral, na presença ou na ausência de camadas de composição mineral diferentes, os urólitos podem ser classificados em simples, mistos ou compostos. São classificados como simples os urólitos com predomínio de um mesmo mineral (maior ou igual a 70\%) em uma ou duas de suas camadas, compostos quando apresentam $70 \%$ ou mais de dois ou mais minerais distintos em camadas diferentes e mistos aqueles que apresentam camadas com diversos tipos de minerais, sem que haja a predominância de um único mineral (composição menor que 70\%) (ULRICH et al., 2008).

Vários métodos podem ser utilizados para avaliar a composição dos urólitos: dois métodos por estimativa (baseando-se no aspecto macroscópico do urólito ou em exames laboratoriais e de imagem associados aos dados da resenha) e dois métodos de análise (qualitativo e quantitativo) (BOVEE \& McGUIRE, 1984; OSBORNE et al., 1989; KOEHLER et al., 2008). Camargo (2004) avaliou 87 urólitos caninos e concluiu que a determinação da composição de urólitos com base em aspectos macroscópicos não deve ser empregada, dada a grande margem de erro (obteve 19,1\% de acerto, em comparação com o método qualitativo).

A análise qualitativa não permite a determinação percentual dos diferentes minerais presentes (ULRICH et al., 1996) e frequentemente falha na detecção de sílica (OSBORNE et al., 1999a; KOEHLER et al., 2008), xantina (KOEHLER et al., 2008), cistina (BOVEE \& McGUIRE, 1984), fármacos (ULRICH et al., 1996) e de componentes que compreenderem menos de 20\% da amostra (RUBY \& LING, 1986). Já os métodos físicos (análise quantitativa) permitem maior exatidão na identificação e quantificação das substâncias e disposição dentro dos urólitos e fornecem melhores informações diagnósticas, prognósticas e terapêuticas (BOVEE \& McGUIRE, 1984; OSBORNE et al., 1989; KOEHLER et al., 2008). A análise quantitativa permite classificar os urólitos em simples, mistos ou compostos, além de diferenciar os diversos subgrupos minerais (por exemplo, oxalato de cálcio monohidratado e dihidratado) (RUBY \& LING, 1986; LULICH et al., 2004). Esses métodos incluem: a cristalografia óptica, a espectroscopia infravermelha, a difratometria do raio$\mathrm{x}$, as técnicas dispersivas de energia (EDAX) e outros (BOVEE \& McGUIRE, 1984; ULRICH et al., 1996).

No presente trabalho, avaliou-se a composição mineral, pelos métodos de análise quantitativa, de urólitos obtidos de cães atendidos no Hospital Veterinário da Faculdade de Medicina Veterinária e Zootecnia da Universidade de São Paulo 
(FMVZ-USP), com objetivo de obter informações que pudessem auxiliar no entendimento da patogenia da urolitíase em cães e, assim, obter subsídios para melhor preconização de terapia e prognóstico.

\section{MATERIAL E MÉTODOS}

Foram avaliados urólitos de 156 cães com diagnóstico de urolitíase (94 machos e 62 fêmeas), com e sem definição racial, atendidos no Hospital Veterinário da FMVZ-USP, durante o período de fevereiro de 1999 a janeiro de 2007. Os urólitos foram obtidos por micção espontânea, por sondagem uretral ou ainda retirados após intervenção cirúrgica. Metade das amostras ( $n=78)$ foi obtida da vesícula urinária; $1,3 \%$ das amostras $(n=2)$ foram obtidas da bexiga e do ureter; 33,9\% $(n=53)$, da bexiga e uretra; $13,5 \%(n=21)$, apenas da uretra; e $1,3 \%(n=2)$, de urólitos renais.

Após lavados e embalados, os urólitos foram enviados para avaliação no Minnesota Urolith Center, na Universidade de Minnesota, St. Paul, MN, EUA. A análise quantitativa foi realizada de acordo com a metodologia empregada no referido centro, que se baseia nos métodos de microscopia de luz polarizada, espectroscopia infravermelha e espectroscopia por raios-X com dispersão de energia (EDAX).

Foram classificados como simples os urólitos com predomínio de um mesmo mineral (maior ou igual a 70\%) em uma ou duas de suas camadas e como compostos quando apresentaram $70 \%$ ou mais de dois ou mais minerais distintos em camadas diferentes. Os urólitos mistos apresentaram quantidades inferiores a 70\% de diversos minerais em suas camadas (ULRICH et al., 2008). Neste estudo, as composições minerais de oxalato de cálcio monohidratado ou de oxalato de cálcio dihidratado foram classificados como oxalato de cálcio. O termo urato inclui urato ácido de amônio, de sódio e ácido úrico, e as formas carbonato, apatita e brushita foram classificadas como fosfato de cálcio.

\section{RESULTADOS}

Os 156 urólitos foram classificados em simples, mistos ou compostos, e houve predomínio de urólitos simples (79,5\%, $\mathrm{n}=124)$. Destes, 47,6\% (59 de 124) eram estruvita e $37,9 \%(n=47)$ oxalato de cálcio. Em menor frequência, urólitos de urato representaram $12,1 \%$ dos urólitos simples $(n=15), 1,6 \%(n=2)$ de fosfato de cálcio e $0,8 \%(n=1)$ de sílica. Todos os urólitos mistos (2,5\%; 4 de 156) continham estruvita e fosfato de cálcio.

Foram classificados como compostos 28 urólitos (18\%). A estruvita foi o mineral mais frequente nas camadas núcleo e pedra; e o fosfato de cálcio, na parede e nos cristais de superfície (Figura 1). Vinte e dois urólitos compostos (78,7\%) não eram passíveis de dissolução parcial, pois apresentavam minerais insolúveis em sua camada externa (oxalato de cálcio e fosfato de cálcio), dois dos cálculos compostos $(7,1 \%)$ eram passíveis de dissolução parcial (camadas externas passíveis de dissolução - estruvita e urato, porém camadas internas não passíveis de dissolução - oxalato de cálcio e fosfato de cálcio), dois dos 28 urólitos compostos $(7,1 \%)$ eram passíveis de dissolução, porém contendo minerais de diferentes solubilidades (urato e estruvita) e, ainda, dois urólitos (7,1\%) continham minerais não passíveis de dissolução (oxalato de cálcio e fosfato de cálcio).

Os cálculos simples de oxalato de cálcio foram observados em 47 cães (idade entre 33 e 228 meses; média 103,3), tendo maior incidência em machos $(n=40)$ que em fêmeas (n=7). Quanto à definição racial, animais sem raça definida $(n=11)$ foram os mais acometidos, seguidos pelos animais das raças Schnauzer e Poodle $(\mathrm{n}=8)$, Pinscher $(\mathrm{n}=7)$, Yorkshire $(\mathrm{n}=4)$ e Dachshund $(\mathrm{n}=2)$ e Bichon Frisé $(\mathrm{n}=2)$. Já urólitos simples de estruvita foram identificados em 59 cães (idade entre 10 e 178 meses; média 73,2), acometendo tanto machos $(\mathrm{n}=30)$, quanto fêmeas $(n=29)$. A raça Poodle foi a mais acometida $(n=14)$, seguida de cães sem raça definida $(\mathrm{n}=11)$, $\operatorname{Cocker}(\mathrm{n}=8)$, Schnauzer $(\mathrm{n}=5)$ e Yorkshire $(\mathrm{n}=5)$. Os urólitos simples de urato $(n=15)$ ocorreram com maior frequência em cães das raças Dálmata $(\mathrm{n}=10)$, cães sem raça definida $(\mathrm{n}=1)$, Cocker $(\mathrm{n}=1)$, Bichon Frisé $(\mathrm{n}=1)$, Fox Terrier $(\mathrm{n}=1)$ e Poodle $(\mathrm{n}=1)$; além de muito mais frequente em machos $(n=13)$ que em fêmeas $(n=2)$.

\section{DISCUSSÃO}

No presente estudo, o predomínio do mineral estruvita corrobora os dados já relatados na literatura (OSBORNE et al., 1986b; OSBORNE et al., 1999c). Entretanto, também é descrito na literatura que estão ocorrendo mudanças quanto à frequência do tipo de composição mineral de urólitos nos cães desde o início da década de 1980. Estudos publicados em vários países indicam o crescente aumento da prevalência de urólitos com oxalato de cálcio, o que coincide com a 


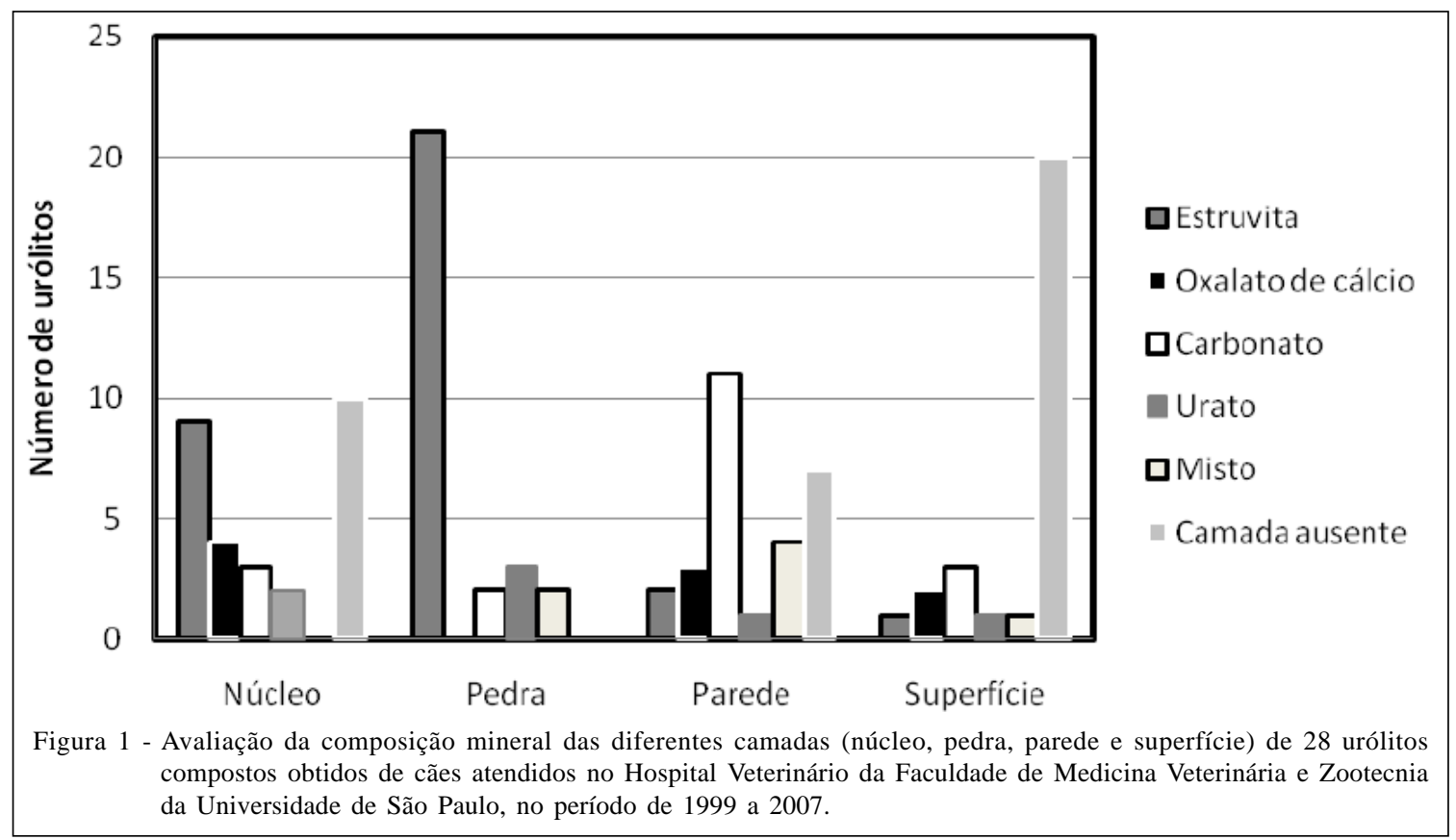

diminuição de urólitos de estruvita (OSBORNE et al., 1989; LEKCHAROENSUK et al., 2002; HOUSTON et al., 2004; SOSNAR et al., 2005).

A estruvita foi detectada em 59 cães (47,6\%) com urólitos simples, em todos os urólitos mistos ( $\mathrm{n}=4$; $100 \%$ ) e também houve predomínio (maior ou igual a $70 \%$ ) em pelo menos uma das camadas de 25 urólitos compostos (89,3\%). Entretanto, também foi observado que o oxalato de cálcio foi a segunda composição mais frequente dos urólitos simples ( $\mathrm{n}=47 ; 37,9 \%)$. A mudança das composições minerais surgiu, provavelmente, em decorrência da introdução de medidas terapêuticas mais eficazes para dissolução de urólitos de estruvita. A diminuição da ocorrência de urólitos de estruvita coincide com a difusão e o uso crescente de dietas designadas para sua dissolução (OSBORNE et al., 1989). O controle de alguns fatores de risco para formação de urólitos de estruvita (como diminuição do pH urinário e redução dos níveis de magnésio e fósforo na dieta) pode predispor a formação dos urólitos de oxalato de cálcio (LULICH et al., 1999). Além disso, o avanço nas estratégias terapêuticas e o desenvolvimento de melhores fármacos antimicrobianos para controle da infecção do trato urinário (UTI) podem contribuir para a diminuição da frequência de urólitos de estruvita. No presente estudo, apesar do predomínio de urólitos simples de estruvita, já se observa também uma frequência relativamente próxima de casos de urólitos de oxalato de cálcio, suscitando-se, assim, a possibilidade sugerida quanto à introdução de dietas terapêuticas no tratamento dos cálculos de estruvita e que poderia favorecer o desenvolvimento de um meio químico para a formação de outros tipos de cristais. Entretanto, não foram identificadas as correlações entre tipo de dieta e composição mineral dos urólitos.

Em relação aos urólitos compostos, também foi identificado o predomínio de estruvita nas camadas núcleo e pedra (em nove e 21 urólitos, respectivamente). Já na camada parede houve um predomínio de fosfato de cálcio em 11 urólitos, oxalato de cálcio em três e estruvita em apenas dois. Na superfície, também houve um predomínio de fosfato de cálcio, seguido de oxalato de cálcio e estruvita. Uma justificativa plausível, para o predomínio de outros minerais e não de estruvita nas camadas mais externas do urólito, poderia ser o uso de dietas terapêuticas na tentativa de dissolver urólitos de estruvita, ocasionando uma redução do pH urinário e de alguns fatores protetores, como magnésio e fósforo e, assim, predispor a precipitação de outros cristais, como oxalato de cálcio. No presente estudo, esses dados não puderam ser compilados individualmente nos casos clínicos.

Além disso, o uso concomitante de antibioticoterapia, na erradicação de bactérias produtoras de urease, também pode reduzir a precipitação de cristais de estruvita. É comum ocorrer a associação das composições minerais de estruvita e fosfato de cálcio na formação de urólitos, já que ambos se formam no mesmo ambiente, ou seja, em urina alcalina em que ocorre a redução da solubilidade de ambos os sais e a concentração aumentada de fósforo predispõe 
a formação de ambos os tipos de urólitos (OSBORNE et al., 1999c).

Portanto, o entendimento e o conhecimento da composição mineral de cada camada do urólito, como também a quantidade presente de cada mineral, são informações importantes para a preconização da terapia apropriada. Exemplo seria a terapia de dissolução que pode ser ineficaz caso o urólito seja composto de minerais com solubilidades distintas ou se um mineral não passível de dissolução envolver completamente ou compreender $20 \%$ ou mais da camada mais externa (ADAMS \& SYME, 2005). No presente estudo, foram observados dois pacientes que apresentaram cálculo composto e que as camadas mais externas do urólito eram passíveis de dissolução (estruvita ou urato), porém camadas mais internas eram compostas de oxalato de cálcio ou fosfato de cálcio que não dissolvem com terapia dietética. Um processo provável para a formação de uma camada externa de estruvita seria o de que urólitos podem lesionar o uroepitélio e predispor a ITU que favorece a precipitação de cristais de estruvita, caso microorganismos produtores de urease estejam envolvidos. Outros dois pacientes apresentaram urólitos com camadas passíveis de dissolução (estruvita e urato), porém a recomendação de terapia seria distinta, reforçando a teoria de que a urina que circunda o urólito, o seu grau de saturação e o tipo de cristalóides calculogênicos formados podem se alterar ao longo do tempo. O conhecimento dos mecanismos de formação do urólito e de todos os fatores que alteram sua formação é essencial para o planejamento de tratamento adequado e a prevenção de possíveis falhas no tratamento. Além disso, tais observações reafirmam a necessidade de acompanhamento periódico durante a terapia de dissolução, pois os fatores que predispõem a formação de um tipo mineral podem ser alterados, e a precipitação de outros tipos minerais pode ser favorecida.

O núcleo do urólito deve ser analisado separadamente sempre que possível, pois as anormalidades subjacentes da formação do urólito podem ser sugeridas pela composição do núcleo. Além disso, fatores que iniciaram a formação do urólito podem ser diferentes da causa de seu crescimento (OSBORNE et al., 1981; OSBORNE et al., 1986a). Assim, são recomendados protocolos de prevenção de minerais que compõem o núcleo, pois a concentração de minerais necessários para formação de urólitos na ausência de um substrato sólido (nucleação homogênea) é superior à requerida para precipitar ao redor de um sólido preexistente (LULICH \& OSBORNE, 2000).

A alta frequência de oxalato de cálcio encontrado em determinadas raças, como Schnauzer miniatura, Lhasa Apso, Yorkshire Terrier, Bichon Frisé, Shih Tzu e Poodle miniatura, corrobora a hipótese de que alguns dos fatores que promovem a formação desse tipo de cálculo são hereditários ou familial (LULICH et al, 1999). Observou-se, no presente estudo, um maior número de cães sem raça definida $(\mathrm{n}=11)$ acometidos por urólitos simples de oxalato de cálcio, seguidos por cães Schnauzer e Poodle ( $\mathrm{n}=8)$. Exceto cães sem raça definida, os resultados encontrados foram semelhantes aos descritos na literatura. Porém, neste estudo, cães mestiços representaram 20,5\% de todos os animais avaliados, seguidos por Poodle (18\%) e Schnauzer (10,9\%); entretanto, não se sabe quanto ao impacto que esses animais mestiços têm sobre a população atendida no hospital.

Urólitos de estruvita também são frequentemente relatados em Schnauzers. Nessa raça acredita-se que haja uma susceptibilidade à infecção do trato urinário em razão de uma anormalidade de defesa local (OSBORNE et al., 1986b). A etiopatogênese desse tipo de urólito tem sido muito estudada, e a infecção do trato urinário (ITU) é um fator importante que predispõe a sua formação. Portanto, pode ser encontrada em qualquer raça, como Poodle, Bichon Frisé, Cocker Spaniel, Lhasa Apso e também em cães sem raça definida (ADAMS \& SYME, 2005). Os resultados referentes às raças dos animais com urólitos simples de estruvita foram semelhantes aos descritos na literatura.

Dos 47 urólitos simples de oxalato de cálcio, 40 foram obtidos de machos e apenas sete de fêmeas, achados condizentes com a literatura, pois os machos podem ser mais predispostos a formação do referido tipo de cálculo urinário por apresentarem menor excreção urinária de citrato, que é um inibidor da formação de cristais, que ocorre em decorrência da menor concentração de estrógeno circulante quando comparada com as fêmeas. (LULICH et al, 1999). Já a estruvita foi encontrada, tanto em fêmeas $(n=29)$, quanto em machos $(n=30)$. Historicamente, fêmeas são muito mais acometidas por esse tipo mineral, já que também são mais predispostas à ITU; por outro lado, a introdução de dietas de dissolução e a uretra mais curta e de maior diâmetro permitem que as fêmeas expilam pequenos cálculos mais facilmente que os machos. Quanto aos urólitos simples de urato $(n=15)$, cães machos $(n=13)$ da raça Dálmata $(n=10)$ foram os mais acometidos. Os achados condizem com a predisposição racial, pois na referida raça ocorre maior excreção urinária de ácido úrico. Além disso, cães de outras raças também podem apresentar maior excreção de ácido 
úrico em decorrência de doenças hepáticas e assim favorecer a formação desse tipo de urólito (BARTGES et al., 1999; GRAUER, 2003; ALBASAN et al., 2005).

O aumento da disponibilidade de protocolos terapêuticos que visam a dissolver os cálculos urinários, bem como a preconização de terapia preventiva, indica que a análise adequada do urólito é essencial para a utilização correta dessas recomendações. Vale ressaltar que avaliações periódicas também são necessárias, pois medidas adotadas para prevenção ou dissolução de alguns tipos de cálculos podem predispor a formação de outros.

\section{CONCLUSÕES}

O presente estudo demonstrou que a análise quantitativa do urólito é fundamental, uma vez que permitiu a classificação em urólitos simples, mistos ou compostos. Caso a análise fosse realizada apenas pelo método qualitativo, essa avaliação seria prejudicada, pois é essencial identificar as similaridades ou diferenças entre os tipos minerais que compõe cada camada dos urólitos. A análise da região central do urólito, ou seja, núcleo ou pedra, merece atenção especial, já que esta pode indicar anormalidades subjacentes ao início da formação do urólito e que deve ser corrigida, quando possível. Os protocolos de dissolução ou prevenção devem ser planejados com base no conhecimento da análise quantitativa dos urólitos.

\section{COMITÊ DE ÉTICA E BEM-ESTAR ANIMAL}

Projeto aprovado sob o n. 809/2005 pela Comissão de Bioética da Faculdade de Medicina Veterinária e Zootecnia da Universidade de São Paulo.

\section{AGRADECIMENTOS}

Aos Serviços de Clínica Médica de Pequenos Animais, de Cirurgia e de Diagnóstico por Imagem do Hospital Veterinário da FMVZ-USP; ao Minnesota Urolith CenterUniversity of Minnesota; à Hill's Pet Nutrition e à Fundação de Amparo à Pesquisa do Estado de São Paulo (processo n. 05/ 57483-7).

\section{REFERÊNCIAS}

ADAMS, L.G.; SYME, H.M. Canine lower urinary tract diseases. In: ETTINGER, S.J.; FELDMAN, E.C. Textbook of veterinary internal medicine. St. Louis: Elsevier, 2005. V.2, p.1850-1874.

ALBASAN, H. et al. Evaluation of the association between sex and risk of forming urate uroliths in Dalmatians. Journal of the American Veterinary Medical Association, v.227, n.4, p.565569, 2005. Disponível em: <http://avmajournals.avma.org/doi/ abs/10.2460/javma.2005.227.565>. Acesso em: 21 set. 2009. doi: 10.2460/javma.2005.227.565.
BARTGES, J.W. et al. Canine urate urolithiasis: etiopathogenesis, diagnosis and management. Veterinary Clinics of North America: Small Animal Practice, v.29, n.1, p.161-191, 1999.

BOVEE, K.C.; MCGUIRE, T. Qualitative and quantitative analysis of uroliths in dogs: definitive determination of chemical type. Journal of the American Veterinary Medical Association, v.185, n.9, p.983-987, 1984.

CAMARGO, C.P. Aspectos clínicos e epidemiológicos de urolitíases em cães e gatos assistidos pelo serviço de nefrologia e urologia da UNESP de Jaboticabal. 2004. 62f. Dissertação (Mestrado em Clínica Médica Veterinária) Faculdade de Ciências Agrárias e Veterinárias, Universidade Estadual Paulista Júlio de Mesquita Filho, Jaboticabal, SP.

GRAUER, G.F. Canine urolithiasis. In: NELSON, R.W.; COUTO, C.G. Small animal internal medicine. St Louis: Mosby, 2003. p.631-641.

HOUSTON, D.M. et al. Canine urolithiasis: a look at over 16.000 urolith submissions to the canadian veterinary urolith centre from February 1998 to April 2003. Canadian Veterinary Journal, v.45, n.3, p.225-230, 2004.

LEKCHAROENSUK, C. et al. Associations between dietary factors in canned food and formation of calcium oxalate uroliths in dogs. American Journal of Veterinary Research, v.63, n.2, p.163169, 2002. Disponível em: <http://avmajournals.avma.org/doi/ abs/10.2460/ajvr.2002.63.163>. Acesso em: 21 set. 2009. doi: 10.2460/ajvr.2002.63.163.

LULICH, J.P.; OSBORNE, C.A. Compound uroliths: treatment and prevention. In: BONAGURA, J.D. Kirk's current veterinary therapy XIII small animal practice. Philadelphia: Saunders, 2000. p.874-877.

KOEHLER, L.A. et al. Canine uroliths: frequently asked questions and their answers. Veterinary Clinics of North America: Small Animal Practice, v.39, p.161-181, 2008. Disponível em: <http://linkinghub.elsevier.com/retrieve/pii/ S0195-5616(08)00161-7>. Acesso em: 13 set. 2009. doi: 10.1016/j.cvsm.2008.09.007.

LULICH, J.P. et al. Distúrbios do trato urinário inferior dos caninos. In: ETTINGER, S.J.; FELDMAN, E.C. Tratado de medicina interna veterinária. Rio de Janeiro: Guanabara Koogan, 2004. V.2, p.1841-1877.

LULICH, J.P. et al. Epidemiology of canine calcium oxalate uroliths. Identifying risk factors. Veterinary Clinics of North America: Small Animal Practice, v.29, n. 1, p.113-122, 1999.

OSBORNE, C.A.; CLINTON, C.W. Urolithiasis. terms and concepts. Veterinary Clinics of North America: Small Animal Practice, v.16, n.1, p.3-17, 1986.

OSBORNE, C.A. et al. Comparison of qualitative and quantitative analyses of canine uroliths. Veterinary Clinics of North America: Small Animal Practice, v.16, n.2, p.317323, 1986a.

OSBORNE, C.A. et al. Canine silica urolithiasis. Risk factors, detection, treatment, and prevention. Veterinary Clinics of North America: Small Animal Practice, v.29, n.1, p.213230, 1999a. 
OSBORNE, C.A. et al. Canine struvite urolithiasis: problem and their dissolution. Journal of the American Veterinary Medical Association, v.179, n.3, p.239-244, 1981.

OSBORNE, C.A. et al. Etiopathogenesis of canine struvite urolithiasis. Veterinary Clinics of North America: Small Animal Practice, v.16, n.1, p.67-86, 1986 b.

OSBORNE, C.A. et al. Medical dissolution and prevention of canine struvite urolithiasis - twenty years of experience. Veterinary Clinics of North America: Small Animal Practice, v.29, n.1, p.73-111, 1999c.

OSBORNE, C.A. et al. Analysis of 77.000 canine uroliths. Perspectives from the Minnesota Urolith Center. Veterinary Clinics of North America: Small Animal Practice, v.29, n.1, p.17-38, 1999b.

OSBORNE, C.A. et al. Relationship of nutritional factors to the cause, dissolution, and prevention of canine uroliths. Veterinary Clinics of North America: Small Animal Practice, v.19, n.3, p.583-619, 1989.
RUBY, A.L.; LING, G.V. Methods of analysis of canine uroliths. Veterinary Clinics of North America: Small Animal Practice, v.16, n.2, p.293-301, 1986.

SENIOR, D.F.; FINLAYSON, B. Initiation and growth of uroliths. Veterinary Clinics of North America: Small Animal Practice, v.16, n.1, p.19-26, 1986.

SOSNAR, M. et al. Epidemiology of canine urolithiasis in the Czech Republic from 1997 to 2002. Journal of Small Animal Practice, v.46, n.4, p.177-184, 2005. Disponível em: <http:/ /www3.interscience.wiley.com/journal/118736620/ abstract?CRETRY=1\&SRETRY=0>. Acesso em: 21 set. 2009. doi: 10.1111/j.1748-5827.2005.tb00308.x

ULRICH, L.K. et al. Urolith analysis: submission, methods, and interpretation. Veterinary Clinics of North America: Small Animal Practice, v.26, n.2, p.393-400, 1996.

ULRICH, L.K. et al. Changing paradigms in the frequency and management of canine compound uroliths. Veterinary Clinics of North America: Small Animal Practice, v.39, p.41-53, 2008. Disponível em: <http://linkinghub.elsevier.com/retrieve/ pii/S0195-5616(08)00163-0>. Acesso em: 13 set. 2009. doi: 10.1016/j.cvsm.2008.09.009. 